Rabaska

Revue d'ethnologie de l'Amérique française

\title{
Patrimoine et patrimonialisation : entre le matériel et l'immatériel
}

\section{Ève-Lyne Cayouette-Ashby}

Volume 5, 2007

URI : https://id.erudit.org/iderudit/019037ar

DOI : https://doi.org/10.7202/019037ar

Aller au sommaire du numéro

Éditeur(s)

Société québécoise d'ethnologie

ISSN

1703-7433 (imprimé)

1916-7350 (numérique)

Découvrir la revue

Citer ce compte rendu

Cayouette-Ashby, È.-L. (2007). Compte rendu de [Patrimoine et patrimonialisation : entre le matériel et l'immatériel]. Rabaska, 5, 126-131.

https://doi.org/10.7202/019037ar d'utilisation que vous pouvez consulter en ligne.

https://apropos.erudit.org/fr/usagers/politique-dutilisation/ 


\section{Colloque}

\section{Patrimoine et patrimonialisation : entre le matériel et l'immatériel}

Ève-Lyne CAyouette-Ashby

Université du Québec à Montréal

Les $1^{\text {er }}$ et 2 décembre 2006, se tenait, dans les locaux de 1'Université Laval, le colloque Patrimoine et patrimonialisation : entre le matériel et l'immatériel. Cette deuxième édition de la Rencontre internationale des jeunes chercheurs en patrimoine rassemblait plus d'une trentaine de chercheurs issus de divers horizons. Ainsi, du Canada à la France, en passant par l'Afrique et la Polynésie française, pour ne citer que ces quelques exemples, les chercheurs invités nous ont présenté un portrait assez global des études actuelles en patrimoine.

Tel que l'indique le titre du colloque, cette rencontre avait pour but d'aborder les liens qui unissent deux volets du patrimoine : le matériel et l'immatériel. En effet, malgré leur caractère indissociable, ces deux dimensions sont trop souvent traitées indépendamment l'une de l'autre et les communications présentées lors de ce colloque ont efficacement démontré la relation étroite qui les unit. Afin d'articuler le débat, le thème principal avait été segmenté en quatre axes : l'objet, le sens, les pratiques et les enjeux. Reprenant cette division, les communications étaient réparties en quatre ateliers.

\section{Premier atelier : Quête d'objets}

Le premier atelier, Quête d'objets, était présidé par Lucie K. Morisset de l'UQÀm et Guy Mercier de l'Université Laval. Avec des sujets tels que la fantomatique dans les représentations de l'espace urbain, le patrimoine et l'aménagement du territoire ou la problématique de la mise en scène muséographique d'un patrimoine immatériel, les chercheurs ont planté le décor de ce colloque dès le départ, en démontrant l'élargissement constant du champ d'étude du patrimoine.

Céline Verguet, doctorante en anthropologie à l'Université Nice SophiaAntipolis, a ouvert le bal en présentant une communication qui traitait de la part de fantomatique dans les représentations de l'espace urbain. Partant de 
l'idée qu'il existe de nombreuses références à des constructions disparues dans les études urbaines, Céline Verguet a démontré l'importance de prendre ces constructions en compte puisqu'elles participent à l'architectonie mentale de l'espace urbain et à son organisation architecturale.

Avec « Politiques incendiaires, patrimoine en fumée : le cas de J.E. Giguère Tabac Ltée », Jocelyn Gadbois, candidat à la maîtrise en ethnologie de l'Université Laval, a soulevé le problème de la disparition de patrimoines suite à la création de nouvelles législations. L'argument principal étant que les nouvelles lois antitabac entraînent une marginalisation de certaines expressions du patrimoine culturel immatériel, Jocelyn Gadbois a développé une réflexion sur le rôle de l'État quant à la protection du patrimoine immatériel.

Depuis plusieurs années, Pierre Bertoncini étudie l'art du graffiti. Dans le cadre d'un stage postdoctoral qu'il effectue en partenariat avec le Musée des civilisations de l'Europe et de la Méditerranée, il s'intéresse aux conditions de patrimonialisation des graffitis bombés. Ainsi, plus que le graffiti, ce sont les enjeux identitaires liés à ce mode de marquage du territoire qui se trouvent au cœur de ses réflexions ainsi que les méthodes de conservation de traces matérielles de ces graffitis éphémères et immatériels.

Pour leur part, Barbara Julien et Yona Jébrak, toutes deux doctorantes en études urbaines à l'Université du Québec à Montréal, nous ont présenté un cas de patrimonialisation d'une reconstruction de guerre. Le district d'Hydrostone à Halifax a été reconstruit suite à une explosion survenue pendant la Première Guerre mondiale. Pourtant, ce fait historique important est passé sous silence par le gouvernement canadien qui a fait de ce lieu un lieu historique national pour le caractère novateur des nouvelles constructions des années 1920.

Doctorant en géographie des universités Laval et Bordeaux, Richard Desnoilles a présenté une communication sur la recherche de la cité idéale. En comparant Québec et Bordeaux, il affirme que ces deux villes patrimoniales reconnues par l'UNESCO utilisent leur patrimoine en tant que vecteur fondamental de la construction de leur image. L'objectif principal de cette communication était de démontrer que les politiques urbaines de ces villes patrimoniales constituent un projet de société en devenir.

La dernière communication de cet atelier a été présentée par Catherine Séguin qui est candidate à la maîtrise en études urbaines à l'Université du Québec à Montréal. Ses recherches portent sur le quartier de Saint-Henri à Montréal et sur les causes de l'annihilation d'une part de sa personnalité. S'appuyant sur l'exemple de la Place Saint-Henri et du square Jacques-Cartier, qui étaient autrefois au centre du paysage de Saint-Henri, Catherine Séguin 
démontre l'échec de nombreux projets de revitalisation urbaine qui ne tiennent pas compte de la mémoire des lieux.

\section{Deuxième atelier : Recherche de sens}

L'atelier Recherche de sens, présidé par Philippe Dubé de l'Université Laval et Réginald Auger du CÉLAT, faisait le pont entre le matériel et l'immatériel. Ainsi, il se voulait un lieu d'étude de la construction et de l'investissement de sens dont les divers patrimoines font l'objet.

L'après-midi a donc débuté avec un détour en Afrique de l'Ouest proposé par Ana Lucia Araujo, doctorante en histoire à l'Université Laval. Ses recherches portent sur le patrimoine de la traite négrière dans cette région du globe. En examinant le cas du mémorial de la maison de Francisco Félix de Souza, quelques particularités quant à la conception et aux méthodes africaines de patrimonialisation sont apparues.

Laureline Lagoutte-Katz, doctorante en ethnologie à l'Université de Bretagne occidentale, nous a ensuite transportés en Bretagne afin de traiter de la surreprésentation alimentaire dans l'affiche patrimoniale bretonne. Cette communication a provoqué une réflexion sur la construction indigène d'une identité collective et sur l'idée d'authenticité.

Barry Magrill, doctorant en histoire de l'art de l'University of British Columbia, s'intéresse à la conservation du patrimoine religieux des communautés autochtones de la Colombie-Britannique. Il a démontré que ce patrimoine était hautement menacé par l'absence de législation ou de désignation patrimoniale reconnue et que la seule solution envisageable passait par la consultation des populations autochtones concernées.

Pour sa part, Capucine Lemaître, docteur en histoire de l'art de l'Université de Rennes 2, a présenté une communication qui traitait de la découverte et de la sauvegarde des mosaïques antiques et médiévales en France, au XIX ${ }^{\mathrm{e}}$ siècle. Elle a montré que la redécouverte de ce patrimoine laissé de côté pendant plusieurs siècles a permis de constituer une nouvelle source d'étude et d'élargir le champ des connaissances historiques en la matière.

Finalement, en conclusion de cette première journée de colloque, Peter Coffman a abordé le sujet de la signification sociale des styles architecturaux. Docteur en histoire de l'art de l'Université Queen, il étudie l'architecture des églises de Terre-Neuve et du Labrador. Avec cette communication, il a démontré que l'architecture doit être étudiée à la lumière du contexte des rivalités qui existaient à l'époque entre l'Église d'Angleterre et la population majoritairement catholique. 


\section{Lancement des collectifs et conférence publique}

La journée s'est conclue par le lancement des collectifs Patrimoine et minorités religieuses : richesse et vulnérabilité, dirigé par Marie-Claude Rocher et Marc Pelchat, et Patrimoine et patrimonialisation : du Québec et d'ailleurs, dirigé par Martin Drouin. Alors que le premier aborde la problématique du patrimoine des minorités religieuses au Québec, telle qu'étudiée lors d'un colloque qui s'est tenu à Montréal en mai dernier, le second rassemble des articles de plus d'une quinzaine de chercheurs et traite de l'architecture et de l'aménagement des villes. Finalement, on proposa une conférence publique de Malcolm Thurlby, qui se déroula à l'église Saint Michael et qui avait pour titre «St. Michael's, Sillery, and its place in the Gothic Revival ».

\section{Troisième atelier : Étude des pratiques}

La seconde journée de ce colloque a débuté par l'atelier, Étude des pratiques, présidé par Yves Bergeron de l'UQÀM et Laurier Turgeon de l'Université Laval. Cet atelier était axé sur les usages et les modalités de mise en valeur du patrimoine.

Nolwenn Rannou, docteur en histoire de l'art de l'Université de Rennes 2, a ouvert l'atelier en traitant de la restauration monumentale en province au XIX ${ }^{\mathrm{e}}$ siècle. Sa présentation portait sur l'analyse de l'œuvre de Joseph Bigot en comparaison avec celle de ses contemporains et a démontré l'ampleur des interventions accomplies par des architectes méconnus.

Ensuite, Diane Joly a abordé le thème du défilé de la Saint-Jean-Baptiste, qui est au centre de ses recherches doctorales en histoire de l'art à l'Université du Québec à Montréal. L'objectif principal de sa communication était de rendre compte des premiers élans patrimoniaux qui ont mené à la scénographie du patrimoine immatériel des Canadiens français à Montréal, en 1925.

Stéphane Couture s'intéresse lui aussi à la culture québécoise dans le cadre de sa maîtrise en histoire à l'Université Laval. Il a démontré à quel point son étude, portant sur l'itinéraire historiographique du coureur des bois depuis 1744 à aujourd'hui, est compliquée par la multiplicité des figures de ce personnage présentes dans la littérature.

Doctorant en sciences de la communication à l'Université d'Avignon et des pays de Vaucluse, José Abreu étudie le statut de l'objet et les valorisations économiques dans le cadre de la protection et la sauvegarde du patrimoine immatériel. Sa communication se basait sur la comparaison entre le réseau Économusée du Canada et des politiques de l'Unesco.

Pour sa part, Cindy Morin a présenté une communication sur l'indissociabilité du matériel et de l'immatériel dans la patrimonialisation de l'héritage archéologique autochtone. Plus précisément, elle a abordé le thème 
de la conservation in situ de sites amérindiens au Canada, en utilisant deux études de cas: SGaang Gwaii en Colombie-Britannique et Áísína'pi en Alberta.

Ensuite, Karim Souiah a traité de l'appropriation culturelle du patrimoine architectural de la ville de La Rochelle par la population locale, entre la fin du XIX ${ }^{\mathrm{e}}$ siècle et la fin du $\mathrm{Xx}^{\mathrm{e}}$ siècle. Doctorant en histoire de l'Université de La Rochelle, Karim Souiah s'intéresse au lien réel ou fantasmé entre le patrimoine et l'identité rochelaise.

Ce troisième atelier s'est conclu avec la communication de Juliette Dutour qui poursuit actuellement des études de doctorat en histoire à l'Université Laval en collaboration avec l'École des hautes études en sciences sociales. Celle-ci nous a entretenus du rôle du tourisme dans la conservation des Monuments historiques français dans la première moitié $\mathrm{du} \mathrm{Xx}^{\mathrm{e}}$ siècle.

Quatrième et dernier atelier : Analyse des enjeux

Présidé par Jean-Yves Andrieux de l'Université Rennes 2 et Thierry Bonnot du CNRS, cet atelier a permis aux chercheurs d'aborder les défis à relever dans le futur.

La première communication a été présentée par Fabrice Argounes, doctorant en relations internationales à l'Institut d'études politiques de Bordeaux. Elle traitait de la question de la géopolitique du patrimoine mondial en s'appuyant sur l'exemple de l'UNESCO. La création du patrimoine naturel et culturel et celle du patrimoine culturel immatériel, trente ans plus tard, ont été analysées par rapport aux enjeux politiques de chacune des époques.

Pour sa part, Quang Vinh Dao s'intéresse à la ville d'Ha-nôi dans le cadre de son doctorat en études urbaines à l'Université du Québec à Montréal. Le postulat central de sa communication était que la prédominance des pratiques sur les objets est un facteur identitaire important au sein des sociétés asiatiques. Cette hypothèse était appuyée par l'exemple de la problématique de l'immatérialité du savoir-faire habitant à Ha-nôi.

De son côté, Véronique Dassié, doctorante en anthropologie de l'École des hautes études en sciences sociales, étudie l'incessante repatrimonialisation des arbres du château de Versailles. Sa communication traitait de la coexistence de deux métaphores distinctes entourant ce patrimoine : la métaphore sociopolitique et la métaphore familiale.

Stagiaire post-doctoral relié au CÉLAT et à la Chaire de recherche du Canada en patrimoine urbain, Richard Gauthier étudie le patrimoine ecclésial et les réaménagements d'églises au Québec. L'idée principale de sa présentation était que de nouvelles fonctions pourraient contribuer à la sauvegarde du patrimoine bâti religieux sans entraîner trop de pression sur le culte. 
France Saint-Jean nous a ensuite présenté une communication sur le patrimoine commémoratif des Rébellions à Saint-Denis-sur-Richelieu. Doctorante en histoire de l'art à l'Université du Québec à Montréal, elle s'intéresse à ce sujet en analysant les formes d'interprétation du patrimoine commémoratif rébellien mises en place par le gouvernement.

Nous transportant en Polynésie française, Olivier Ginolin a abordé le sujet de l'identité ma'ohi. Doctorant en anthropologie à l'École doctorale du Musée de l'Homme, il étudie la revalorisation de pratiques artisanales disparues en tant que modes d'expression de la culture ma'ohi populaire contemporaine.

Pour conclure cet atelier, Cynthia Dahomé, doctorante en anthropologie à l'École des hautes études en sciences sociales, nous a offert une communication sur le «choc des altérités » atemporelles à l'Afrique mythique. Le thème central de son discours était celui de l'homogénéisation d'un patrimoine culturel «noir» comme fruit de l'expérience commune de la domination telle que vécue tant par les Antillais que par les Africains.

\section{Conclusion}

Doctorante en ethnologie à l'Université Laval et membre du comité organisateur de cette Deuxième Rencontre internationale des jeunes chercheurs en patrimoine, Marie-Blanche Fourcade a prononcé le mot de la fin, concluant ainsi ces deux journées bien remplies.

Ce colloque aura permis de constater une augmentation de l'intérêt des chercheurs pour le patrimoine immatériel. C'est d'ailleurs en établissant des relations entre ces deux dimensions du patrimoine que l'on peut remettre en contexte les objets patrimoniaux.

Finalement, comme l'a résumé Luc Noppen, la qualité des interventions et la richesse des travaux de recherche présentés lors de cette rencontre en ont fait un franc succès. Ce qu'il faudra retenir de ce colloque, c'est que la relève dans le secteur du patrimoine est manifestement assurée et que l'on assiste à une ouverture des champs de recherche. Bref, nul doute que les rencontres se poursuivront, alimentées par le nombre grandissant de programmes de formation offerts par diverses universités, ce qui démontre que le secteur du patrimoine est en réel développement. 